\title{
A Nearest Neighbor Method Using Bisectors
}

\author{
Mineichi Kudo, Hideyuki Imai, Akira Tanaka, and Tetsuya Murai \\ Division of Systems and Information Engineering \\ Graduate School of Engineering \\ Hokkaido University \\ Kita-13, Nishi-8, Kita-ku, Sapporo 060-8628, Japan \\ \{mine,imai, takira, murahiko\}@main.eng.hokudai.ac.jp \\ http://ips9.main.eng.hokudai.ac.jp
}

\begin{abstract}
A novel algorithm for finding the nearest neighbor was proposed. According to the development of modern technology, the demand is increasing in large-scale datasets with a large number of samples and a large number of features. However, almost all sophisticated algorithms proposed so far are effective only in a small number of features, say, up to 10. This is because in a high-dimensional space many pairs of samples share a same distance. Then the naive algorithm outperforms the others. In this study, we considered to utilize a sequential information of distances obtained by the examined training samples. Indeed, a combinatorial information of examined samples was used as bisectors between possible pairs of them. With this algorithm, a query is processed in $O(\alpha \beta n d)$ for $n$ samples in a $d$-dimensional space and for $\alpha, \beta<1$, in expense of a preprocessing time and space in $O\left(n^{2}\right)$. We examined the performance of the algorithm.
\end{abstract}

\section{Introduction}

The $k$ nearest neighbor $(k-\mathrm{NN})$ method (Cover and Hart, 1967) is very popular in pattern recognition. This method is effective both for estimation of densities and for classification. However, the method requires a large amount of computation to calculate the distances of a query sample to all training samples. To reduce the amount of computation, many methods have been proposed (Hart, 1968; Gates, 1972; Dasarathy, 1994; Chang and Wu, 1993; Fukunaga and Narenda, 1975), especially for classification (Hart, 1968; Gates, 1972; Dasarathy, 1994).

There are two approaches to reduce the computational cost: one group of methods aims to reduce the size of the training sample set to be referred in query stage and another group aims to reduce the number of distance calculations in query stage by adopting an efficient search procedure. In this paper, we focus on the latter approach.

A trial to find a $(1+\epsilon)$ approximate nearest neighbor, Arya et al. (1998) proposed an algorithm which runs in $O(c \log n)$ for answering a query, where $c$ grows exponentially in $d$ and polynomially in $1 / \epsilon$. In addition, Kleinberg (1997) 
proposed two algorithms for the same goal; one runs in $O\left(\left(d \log ^{2} d\right)(d+\log n)\right)$ in query time and another is said to run linear in $d$ asymptotically. Both are able to use to find the nearest neighbor with a sufficiently small value of $\epsilon$. However, in even these algorithms, it is still unclear if they really runs faster than the naive algorithm in very high-dimensional cases. This is because we have to set $\epsilon$ close to zero in the case, and the complexity analysis hides the influence by $1 / \epsilon$. So, we propose an algorithm which runs really faster than the naive algorithm when the distance is measured by Euclidean distance.

\section{Key Ideas}

The key idea to do search efficiently is to use ultimately a sequential information obtained so far. After we examined some points (samples) in distance calculation between them and a query point (sample), we have much information more than the sum of individual distance evidences. In many algorithms, only the most critical evidence, the distance to the current candidate of the nearest neighbor, is used. However, we may use the second and the third candidates as well. In fact, it is possible to consider a sequence of these points examined up to the current stage. We will use one of such information. In this study, we focus on the nearest neighbor, $1-\mathrm{NN}$, instead of $k-\mathrm{NN}$, but the idea is easily extended to $k$-NN cases.

Let us assume that we have examined two points $x_{1}$ and $x_{2}$ and have calculated the distances $d\left(x_{1}, q\right)$ and $d\left(x_{2}, q\right)$, and assume that $d\left(x_{1}, q\right)<d\left(x_{2}, q\right)$. What is the information at this time? Most often used information is that the true nearest neighbor of $q$ has a distance less than $d\left(x_{1}, q\right)$, thus we do not have to check samples with distance larger than this value. However, information like " $x_{1}$ is closer to $q$ than $x_{2}$ " may give us a possibility such as any sample to which $x_{2}$ is closer than $x_{1}$ can omit from the further search. If so, for three points examined so far, we have three same kinds of information, and $\left(\begin{array}{l}4 \\ 2\end{array}\right)=6$ for four points, and so on. Of course, this is not always true. However, under some condition, we can make such a decision.

Under which condition can we use such decisions ? Let us explain it in a two-dimensional case (Fig.1). In Fig.1, $x_{5}$ is the closest point to a query point $q$, that is, $x_{5}$ is the answer, but has not examined. Four points $\left(x_{1}-x_{4}\right)$ are already examined, and six perpendicular bisectors between them are drawn. Here, bisector $b_{13}$ should be out of consideration. This is because this bisector separates the answer $x_{5}$ and $q$ into different regions. With five bisectors, we can conclude that the closest point to $q$ is in the hatched region. In the following, we analyze about which bisectors we can use for narrowing the search area.

\section{$3 \quad$ Effective Bisectors}

It is easy to determine which bisectors are effective and which are not. Here a bisector is said to be "effective" when the bisector dose not separate the answer 


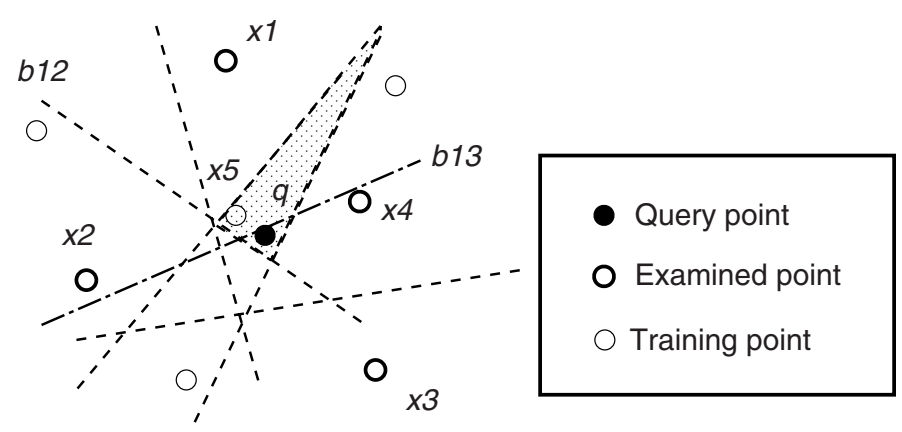

Fig. 1. Narrowing by bisectors. Four of eight samples are already examined and five effective bisectors between these four points are used for narrowing the search area.

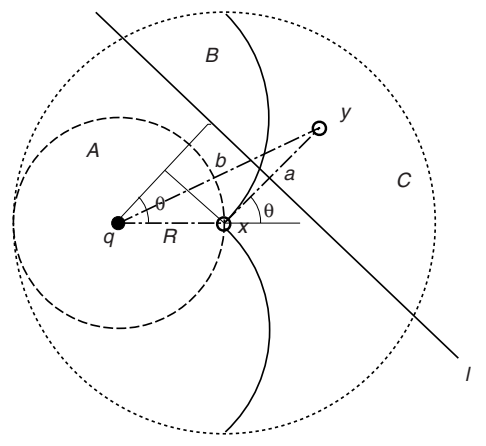

Fig. 2. An effective bisector. Among distinct regions $\mathrm{A}, \mathrm{B}$ and $\mathrm{C}$, point $y$ having an effective bisector must exist in only $\mathrm{C}$.

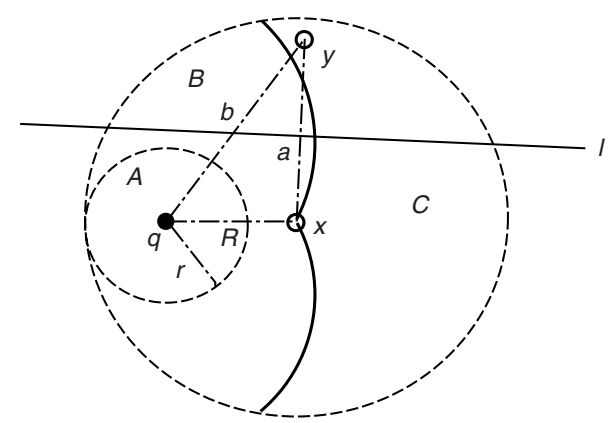

Fig. 3. Extended effective region (C). Here, a solution is assumed in the small ball centered at $q$.

point and the query point into different regions separated by the bisector. An illustrative examples is shown in Fig. 2.

In this figure, two points $x$ and $y$ are already examined ( $x$ is closer to $q$ ) and we know distances $R=d(x, q)$ and $b=d(y, q)$. Let us assume that the distance between $x$ and $y, a=d(x, y)$, is also known. It is enough to search region $\mathrm{A}$ for finding a better candidate than $x$. So, the effective bisectors should not intersect A. Then, the necessary and sufficient condition for the bisector between $x$ and $y$ to be effective is

$$
b^{2} \geq R^{2}+2 R a
$$

\section{(Proof)}

Let us assume that the perpendicular bisector $l$ of $x$ and $y$ does not pass though the ball with radius $R$ centered at $q$. Then following has to be satisfied

$$
\begin{aligned}
a / 2+R \cos \theta & \geq R \\
a & \geq 2 R(1-\cos \theta) .
\end{aligned}
$$


This inequality shows the condition that angle $\theta$ has to satisfied. To translate this angle condition to the distance condition related to $b=d(q, y)$, the following is derived:

$$
\begin{aligned}
b^{2} & =(R+a \cos \theta)^{2}+a^{2} \sin ^{2} \theta \\
& =R^{2}+a^{2}+2 R a \cos \theta \\
& =R^{2}+2 R a(a / 2 R+\cos \theta) \\
& \geq R^{2}+2 R a
\end{aligned}
$$

The last inequality is from (1). Q.E.D.

The boundary equation (1) with equality is called "Cardioid" in geometry. It is noted that region $\mathrm{A}$ in Fig. 2 can be ignored because we impose that $x$ is closer than $y$. If not, exchange $x$ and $y$. So the probability that $y$ occurs in region $\mathrm{C}$ is $P(C) / P(B+C)$, where $P(X)$ is the probability of region $X$. In a uniform distribution, the probability $P(C)$ is about 0.39 for $d=2$ and increases to 0.5 as $d$ increases. However, $P(B)$ grows with dimensionality up to $1-P(C)$. As a result, we may assume that the probability that a new point ( $y$ in this example) generates an effective bisector incorporated with $x$ is $1 / 2$ regardless of dimensionality.

It should be noted that we cannot narrow the search region directly into region $\mathrm{A}$, because knowing whether a sample exists in $\mathrm{A}$ or not is knowing the distance between that point and the query point. On the contrary, with a bisector associated with $x$ and $y$, it is enough to check to which of $x$ and $y$ that sample is closer. This is easily done if we know the distance between any pair of training points. As a result, with preprocessing to calculate the distances of every pair, we can do this efficiently.

In this study, we extend the condition for effective bisectors to another in the situation in which we want to find a sample close to $q$ within distance $r$. Such a query is interesting itself and is able to extend to find the nearest neighbor by repeating this query with several values of $r=r_{1}, r_{2}, \ldots, r_{t}\left(r_{1}<r_{2}<\cdots<\right.$ $\left.r_{t}=\infty\right)$. As will be described later, this brings a benefit in computation cost. It is also possible to set the value of $r$ to the distance $R$ of the current candidate of the nearest neighbor of $q$ in the middle of search. Then, it is guaranteed to find the nearest neighbor.

In this problem setting, for a solution within $r$, the condition under which a bisector $b(x, y)$ is effective in the same setting above is given by

$$
b^{2} \geq R^{2}+2 r a .
$$

This is easy to confirm (Fig. 3). For example, for $r=0$, every bisector is effective. According to a smaller value of $r$, a larger effective region $\mathrm{C}$ is obtained.

\subsection{Algorithm}

The algorithm is shown in Fig. 4. 
Step 0: (Preprocessing)

$n:$ The number of training samples

$q$ : The query sample

$r$ : The search threshold (user-specific)

$t$ : The possible number of $r$ (user-specific)

$s:$ The number of bisectors (user-specific)

$\theta[i] \leftarrow \infty$ : The termination threshold on $x_{i}$

$x_{c}$ : The current nearest neighbor sample

$R \leftarrow \infty$ : The current minimum distance to $q$

For every pair of $x_{i}$ and $x_{j}$

Store the distance $d\left(x_{i}, x_{j}\right)$ in array $d[i, j]$

For $i=1$ to $n$

$\theta[i] \leftarrow d\left(x_{i}, x_{1-N N}^{i}\right) / 2$

where, $x_{1-N N}^{i}$ is the nearest neighbor of $x_{i}$

Procedure main

Step 1 Repeat the following with $r=r_{0}, r_{1}, \ldots, r_{t}=\infty$

$r^{*} \leftarrow r$

For $i=1$ to $n$

Step $2 \quad$ if BallTest is "not passed" then continue with $i \leftarrow i+1$

Step 3 if BisectorTest is "not passed" then continue with $i \leftarrow i+1$

Step $4 \quad$ Calculate distance $d\left(q, x_{i}\right)$

Step 5 Do CreateBisector

Step $6 \quad$ If $d\left(q, x_{i}\right)<R$, update $R \leftarrow d\left(q, x_{i}\right) ; c \leftarrow i ; r \leftarrow \min (r, R)$

Step 7 if TerminationTest is valid then goto Step 8

Step 8 if $R<r^{*}$ then output $x_{c}$, else go to Step 1

Procedure BallTest

if $d[c, i]<R$ return "passed", otherwise return "not passed"

Procedure BisectorTest

For the latest $s$ bisectors $b\left(x_{j}, x_{k}\right)\left(d\left(x_{j}, q\right)<d\left(x_{k}, q\right)\right)$

if $d[i, j]>d[i, k]$ then return "not passed"

return "passed"

Procedure CreateBisector

$x \leftarrow x_{c} ; y \leftarrow x_{i}$

if $d(x, q)>d(y, q)$ then exchange $x \leftrightarrow y$

$R \leftarrow d(q, x) ; a \leftarrow d[i, c] ; b \leftarrow d(q, y)$

if $b^{2} \geq R^{2}+2 r a$ then register $b(x, y)$

Procedure TerminationTest

if $d\left(q, x_{i}\right)<\theta[i]$ then return "valid" else "not valid"

Fig. 4. Algorithm.

The termination condition (Kudo et el., 2003) is also incorporated in Step 7. With the termination condition, we may find the solution in the middle of search and terminate the procedure, but this condition does not work well in highdimensional cases.

In this algorithm, 1) a ball test is carried out in $O(1)$ (Step 2), 2) a bisector test is in $O(2 s)$ (Step 3), 3) a distance calculation in $O(d)$ (Step 4), and 4) a 
construction of a new bisector in $O(1)$. Let us assume that a query sample passes Step 2 in probability $\alpha$ and Step 3 in probability $\beta$. Then the expected cost is $O(n((1-\alpha)+2 s \alpha(1-\beta)+\alpha \beta(d+1))) \simeq O(\alpha \beta n d)$.

\section{Effectiveness of Bisectors}

We conducted a simple experiment to measure to what degree the proposed algorithm is efficient. The samples are generated according to a multivariate Gaussian with a unit covariance and zero mean. The number of samples was varied as $n=1000,5000,10000,20000$ and the dimensionality was varied as $d=2,5,10,20,30,50$. The computational costs can be estimated by how often a query sample passes Step 2 (ball test), Step 3 (bisector test) and Step 4 (distance calculations). So, we counted the number of samples passed these steps. The results are shown in Figs. 5 and 6.

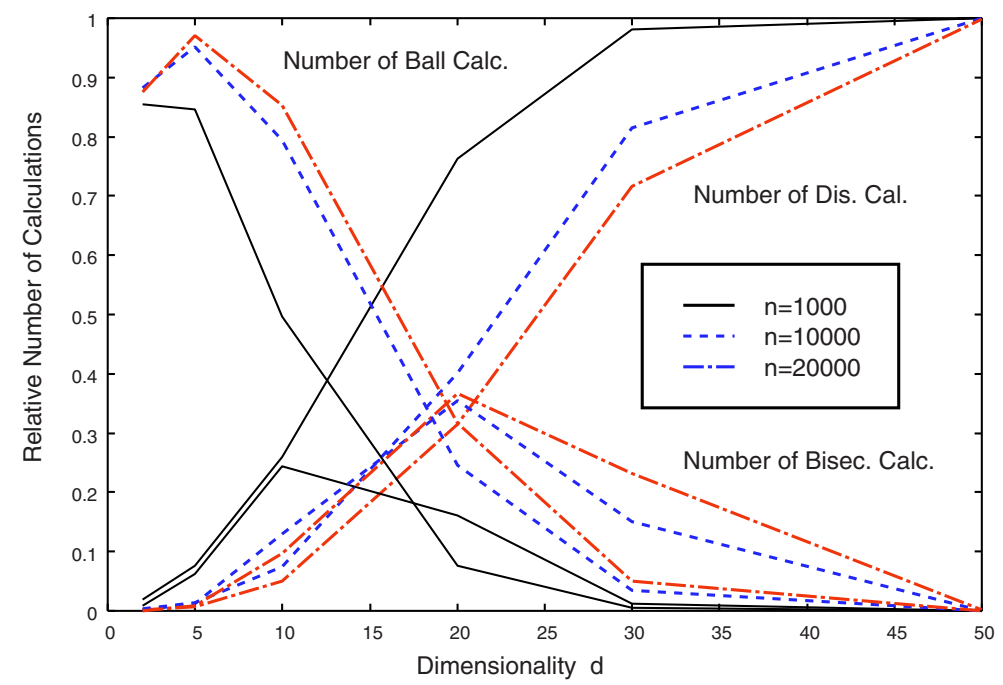

Fig. 5. Relative number of calculations as letting that of the naive algorithm be one.

In Fig. 5, the summand of three curves becomes almost one. If the amount is less than one, it means that the termination condition happened. From this figure, we can see

1. The ball test works well in low-dimensional cases, but decreases exponentially in dimensionality.

2. The bisector test works even up to a moderate size of dimensionality. The effectiveness decreases also as dimensionality increases, but still useful to reduce the number of distance calculations. It looks too low in low-dimensional cases, but this is because the ball test is carried out before the bisector test.

From Fig. 6, it can be read that the relative distance calculation decreases as $n$ increases, which means that the order is less than $O(n d)$. 


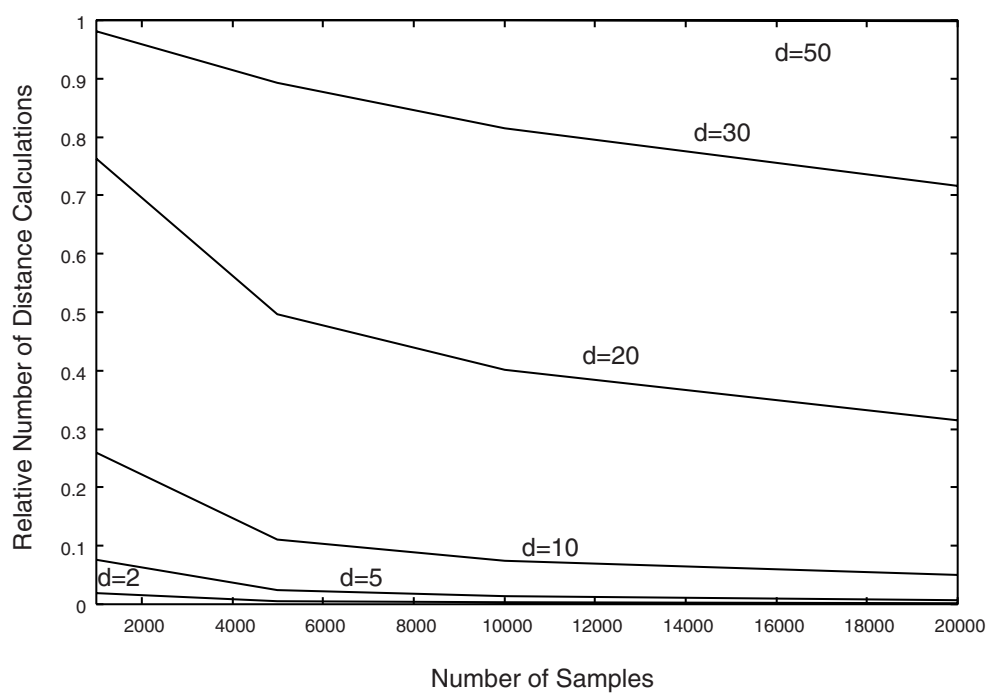

Fig. 6. Relative number of distance calculations as the number of samples increases.

Table 1. Search time (Pentium4, 2GHz, 2GB Memory, $256 \mathrm{~KB}$ cache, Linux). Preprocessing time is not included.

\begin{tabular}{lrrrlll}
\hline Dataset & $C$ & $d n\left(n_{t r}: n_{t e}\right)$ & \multicolumn{3}{c}{ Time $(\mathrm{ms})$} \\
\cline { 5 - 6 } & & & & & Naive Proposed & Ann \\
\hline Sonar & 2 & 60 & $103(8: 2)$ & 0.008 & 0.007 & 0.01 \\
mfeat & 10 & 187 & $2000(8: 2)$ & 0.475 & 0.585 & 0.454 \\
musk & 2 & 166 & $476(8: 2)$ & 0.047 & 0.028 & 0.037 \\
Jap. Char & 30 & 196 & $6000(1: 1)$ & 11.7 & 7.07 & 8.42 \\
\hline
\end{tabular}

Table 2. Probabilities of passing several steps.

\begin{tabular}{llll}
\hline Dataset & $\alpha$ & $\beta$ & $\alpha \dot{\beta}$ \\
\hline sonar & 0.63 & 0.89 & 0.56 \\
mfeat & 0.84 & 0.99 & 0.83 \\
musk & 0.31 & 0.93 & 0.29 \\
Jap. Char & 0.45 & 0.88 & 0.40 \\
\hline
\end{tabular}

\section{Experiments}

We used four real-world datasets of 'sonar', 'mfeat', 'musk' and 'Japanese Characters.' The first three are taken from machine learning databases in UCI (UCI Repository of Machine Learning Databases, 1991). The sample was divided to the training and testing sample sets by $n=n_{t r}+n_{t e}$ as shown in Table 1 . For comparison, ANN (Arya et al. (1998)) was carried out with $\epsilon=0$. The parameter used in the proposed method, $t=3$ and $r_{i}(i=1,3)$ were determined experimentally. In addition, $s$ was set to $d / 2$. The results are shown in Tables 1 and 2. From Table 2, we can see that the probability that training samples pass the ball test, $\alpha$, is lesser than as expected, but the probability that training samples pass the bisector test, $\beta$, is higher than as expected. The highness of $1-\alpha$ can be explained as in these practical problems data form some clusters from their natures. On the other hand, the lowness of $1-\beta$ shows that many training samples share almost a same distance to a query point. This is just the problem that we mentioned first, so, unfortunately, we cannot recognize the 
effectiveness of our way using bisectors. To make clear the reason, we examined further. Then it turned out that the number of found effective bisectors was very small, a few percent of the total number of training samples, in contrast to our analysis $(1 / 2)$, but once such a bisector was found, then it worked well. More precisely, only a few bisectors (less than one percent of total number of training samples, for example, $0.2 \%$ in Japanese Character Database) were contributed the value of $\beta$. Therefore, the probability that effective bisectors were found was not so high, but the found effective bisectors worked very good.

\section{Discussion}

The question to be answered is that this is faster than the previous many algorithms. It requires a preprocessing time of $O\left(n^{2}\right)$ and the same amount of space. It is obvious that the algorithm needs $O(\alpha \beta n d)$ in query time that is less than that of the naive algorithm that needs $O(n d)$, although there is no progress in the definition of large Oh. So, the question is reduced to that $\alpha$ (the probability that training samples pass the ball test) and $\beta$ (the probability that training samples pass the bisector test) is really less than one even in high dimensional cases. The answer was partly yes from the presented experiments.

We can know about the time complexity as follows. Let $p$ be the probability that an effective bisector is found and $q$ be the probability of the area for further search reduced by the bisector. Then, the time complexity $T(n)$ for examining $n$ training samples in $d$ dimension can be written as $T(n)=d+2+p T(q n)+$ $(1-p) T(n-1)$. Then, we can see that $T(n) \leq O(n d)$. The equality holds for $q n=n-1$. Therefore, usually the complexity is better than that of the naive one. To have more precise complexity, we have to take into consideration the influence by successive bisectors.

One more practical issue is the effectiveness of cache. Recent CPUs are equipped with some amount of memory cache, so that the memory access to a sequential data is processed very fast. On the contrary, a random access as the proposed method does, is very slow compared with such a sequential memory access. This make less the advantage of the proposed algorithm. However, for very large datasets, every data should be kept in a disk not in a memory, then the advantage would be alive.

\section{Conclusion}

A novel nearest neighbor algorithm was proposed. It utilized a sequence of information obtained so far. The experimental results seem to support its efficiency even in high dimensional cases in which the previous algorithms are not better than the naive algorithm. The main drawback of $O\left(n^{2}\right)$ space would be reduced by developing the data structure. 


\section{References}

1. T.M. Cover and P.E. Hart, Nearest neighbor pattern classification, IEEE Trans. Information Theory, vol.IT-13, no.1, pp.21-27, Jan. 1967.

2. P.E. Hart, The condensed nearest neighbor rule, IEEE Trans. Information Theory, vol.IT-14, no.3, pp.515-516, May 1968.

3. G.W. Gates, The reduced nearest neighbor rule, IEEE Trans. Information Theory, vol.IT-18, no.3, pp.431-433, May 1972.

4. B.V. Dasarathy, Minimal consistent set(MCS) identification for optimal neighbor decision systems design, IEEE Trans. Systems, Man, \& Cybernetics, vol.SMC-24, no.3, pp.511-517, March 1994.

5. K. Fukunaga and P.M. Narendra, A branch-and-bound algorithm for computing $k$ nearest neighbors, IEEE Trans. Computers, vol.C-24, no.7, pp.750-753, July 1975.

6. C.-C. Chang and T.-C. Wu, A hashing-oriented nearest neighbor searching scheme. Pattern Recognition Letters, vol.14, pp.625-630, August 1993.

7. K. Fukunaga ,Nonparametric Density Estimation, in Introduction to Statistical Pattern Recognition, pp.268-287, Academic Press, 1990.

8. P. M. Murphy and D. W. Aha, UCI Repository of Machine Learning Databases [Machine-Readable Data Repository]. University of California, Department of Information and Computer Science, Irvine, California, 1991.

9. S. Maneewongvatana and D. M. Mount, The Analysis of a Probabilistic Approach to Nearest Neighbor Searching. In Proc. Yth Workshop on Algorithms and Data Structures (WADS 2001),2001, pp. 276-286.

10. S. Arya, D. M. Mount, N. S. Netanyahu, R. Silverman, and A. Y. Wu, An optimal algorithm for approximate nearest neighbor searching, Journal of the ACM,45 (1998), pp. 891-923.

11. J. Kleinberg, Two algorithms for nearest-neighbor search in high dimensions. In Proc. 29th ACM Symposium on Theory of Computing,1997, pp. 599-608.

12. M. Kudo, N. Masuyama and M. Shimbo, Simple termination conditions for knearest neighbor method. Pattern Recognition Letters, 24(2003), 1213-1223. 